\title{
AKA unknown male Foxtrot 23/4: alias assignment for unidentified emergency room patients
}

\author{
Adam J Brooks, Cara Macnab, Ken Boffard
}

\begin{abstract}
Objectives-To introduce a unique system of alias assignment for patients whose identity is initially unknown at time of admission to the emergency unit; to prevent confusion and cases of mistaken identity.

Methods-At the triage area the "unknown" patient is given a "forename" using the phonetic alphabet according to the stage of the current name cycle. The sex of the patient is included as well as the unknown status and a "surname" is added as the numerical date. Thus an unknown male patient admitted on the 24th of April at the start of a new name cycle would be known as "unknown male Alpha 24/4".

Results-Ten thousand alias assignments have been issued to patients since the introduction of the system in 1985.

Conclusion-This system is a simple yet effective, tried and tested method for the unique identification of unknown patients, which allows easy communication and retrieval of data for inquiries.

( $($ Accid Emerg Med 1999;16:171-173)
\end{abstract}

Keywords: alias; unknown patients

All trauma units and casualty departments receive their share of patients whose true identity is unknown at the time of arrival at hospital, either because they are not carrying identity, their condition is too urgent, or because they are too inebriated or obtunded and are therefore not in a position to identify themselves.

Depending on the country or institution in which they find themselves, they are variously identified as "John Doe", "Joe Public" or commonly "unknown male" or "resus 1 ". This is to allow allocation of a hospital number for the immediate processing of laboratory investigations and patient identification for radiography and theatre. However the problem becomes more complex when an emergency department admits more than one unidentified patient at a time. These patients then become known as "unknown male 1 " and "unknown male 2 " or "unknown resus 3" etc.

Unfortunately, this system breaks down when unidentified patients are admitted to the same ward, so that two "unknown male 1 " patients admitted on different days or shifts, end up in the same area potentially sharing blood test and radiography results or even cross matched blood. While safeguards in the form of hospital admission numbers are built in to this system, error can occur. Hospital staff do not think in numerical terms, but are used to names. Some admission department's computers are only able to issue hospital numbers in association with a name, after the arrival of the patient, even when treatment has started. Blood cross matching, etc may have to be delayed in the emergency department pending the details being available. Additionally, inquiries from anxious relatives via the hospital switchboard or admissions department are difficult to correlate with the patients as a computer search of hospital records against the patients name does not pick up an "unknown".

The Johannesburg Hospital Trauma Unit resuscitates 1500 severely injured patients a year and has a further 2500 trauma admissions. Over the years the number of unidentified patients presenting to our institution has increased both because of the population group served and the severity of injuries received, to a point where we receive approximately 800 patients annually whose identity is unknown at the time of admission.

In 1985 we looked at the various ways of identifying patients by name, in the form of assigning an alias to each unidentified patient on arrival. To accommodate those patients who remained unidentified, this alias had to be unique to that patient for the duration of their stay in hospital.

The system requirements were laid down:

(1) It had to be easy to administer.

(2) Identifying alias had to be issued from the triage desk of the emergency department.

(3) The retrieval of the alias both by computer and manually had to be easy to facilitate inquiries.

(4) "Names" had to be easily remembered, such that they could be used in normal conversation or case presentations.

(5) There had to be a complete unique identity for each patient, which would stay with the patient throughout their hospital stay, even after they had been correctly identified or in case they died before absolute identification was made. 
Table 1 Alias assignment

\begin{tabular}{ll}
\hline Alpha & November \\
Bravo & Oscar \\
Charlie & Papa \\
Delta & Quebec \\
Echo & Romeo \\
Foxtrot & Sierra \\
Golf & Tango \\
Hotel & Uniform \\
India & Victor \\
Juliet & Whiskey \\
Kilo & X-Ray \\
Lima & Yankee \\
Mike & Zulu \\
\hline
\end{tabular}

Method

It was decided to base the system on the phonetic alphabet (table 1). This is in common use in radio procedures and therefore the concept was relatively familiar to most personnel. This name was given as the "forename" and therefore provided a pool of 26 easily retrievable identifiers. While there are several phonetic alphabet labels that could appear as real forenames, for example "Charlie", "Victor", the alias was prefixed by "unknown" and the sex of the patient- "unknown male Charlie". Therefore in this context it was felt best to leave the phonetic alphabet unchanged, rather than to increase confusion with unfamiliar phonetic names.

To reduce the chance of shared names further a "surname" was allocated to each patient corresponding to the date of admission. Thus at the beginning of any name cycle, an unidentified male patient who was admitted on the 20th of April (the fourth month) was allocated the alias "unknown male Alpha 20/4". A second and third patient admitted on the same day would be "unknown female Bravo 20/4"

Enter date and sex to confirm alias allocation

\begin{tabular}{|l|l|l|l|l|l|l|l|l|}
\hline & DATE & SEX & DATE & SEX & DATE & SEX & DATE & SEX \\
\hline Alpha & & & & & & & & \\
\hline Bravo & & & & & & & & \\
\hline Charlie & & & & & & & & \\
\hline Delta & & & & & & & & \\
\hline Echo & & & & & & & & \\
\hline Foxtrot & & & & & & & & \\
\hline Golf & & & & & & & & \\
\hline Hotel & & & & & & & & \\
\hline India & & & & & & & & \\
\hline Juliet & & & & & & & & \\
\hline Kilo & & & & & & & & \\
\hline Lima & & & & & & & & \\
\hline Mike & & & & & & & & \\
\hline November & & & & & & & & \\
\hline Oscar & & & & & & & & \\
\hline Papa & & & & & & & & \\
\hline Quebec & & & & & & & & \\
\hline Romeo & & & & & & & & \\
\hline Sierra & & & & & & & & \\
\hline Tango & & & & & & & & \\
\hline Uniform & & & & & & & & \\
\hline Victor & & & & & & & & \\
\hline Whiskey & & & & & & & & \\
\hline X-Ray & & & & & & & & \\
\hline Yankee & & & & & & & & \\
\hline Zulu & & & & & & & & \\
\hline
\end{tabular}

Figure 1 Alias assignment: list kept at entrance to resuscitation room.
HOSP NR: 2394874

ADMITTED: 23/4/98

SMITH JOHN

MR

AKA UNKNOWN MALE FOXTROT 23/4

81 FOURTEEN AVENUE PARKTOWN 2193

$18 / 11 / 65$

32 YEARS

MALE

\section{DR KD BOFFARD}

Figure 2 Sample hospital sticker.

and "unknown male Charlie 20/4". If this patient had been admitted five days later, the alias would have been "unknown male Charlie 25/4". Initially the "surname" had to be entered in letters "unknown female Charlie twentyfive" as the computer was unable to accept numeric "surnames". Shortly after inception of the system the computers were updated to accept numeric terms which allowed the inclusion of the month of admission, allowing greater specificity.

A list (fig 1) was kept at the entrance to the resuscitation room and on arrival of an unidentified patient at hospital, the nurse in charge of the area retrieved the next name from the list and ticked off that it had been allocated by placing the date (that is, "surname") and the sex in the next vacant box. In this way it was possible to keep a visual record of how many names had been allocated over a particular period. The cycle of names starts with Alpha and continues through to Zulu irrespective of the date of admission before the cycle starts again.

When the patient is formally identified, the medical records department is notified and all available data are entered as they become available (for example date of birth, full address, etc). The computer registers the actual name of the patient as the new primary name and the alias as an AKA (also known as) alternate-AKA unknown male Foxtrot 23/4 (fig 2), but allows searches to be made under either name, with all other details merged and the hospital identification number common to both. If the patient has had a previous admission, the previous hospital number is cross referenced but the current number remains in use throughout this and future hospital stays.

\section{Results}

This system has been in use at the Johannesburg Hospital Trauma Unit for 13 years. In this time we have seen a steady increase in the number of unknown patients who are treated in the unit. We estimate that 10000 alias assignments have been issued to patients in this time with 828 allocated in the past 12 months. The greatest number of true unidentified patients we have seen on a single day is 12 .

Medical, nursing, and clerical staff throughout the hospital departments have been happy with the system. As the alias serves as a communication tool and identifier in conjunc- 
tion with a unique hospital number it has been easy to work with and has reduced verbal confusion of patients.

\section{Discussion}

For a system of alias assignment to work it has to be simple to apply and result in less confusion for both patients and staff. Using a combination of the phonetic alphabet, sex, and date we have designed a system with a number of advantages:

(1) The name is specific to the individual and allows easy communication of the identity. There is a pool of more than 19000 individual identifying "names" which is unlikely to be exhausted in most institutions. While the system could break down if more than 26 patients of the same sex were admitted in a single 24 hour period, this is unlikely and has not happened in the 13 years the system has been in use in our hospital despite a large number of multiple casualty incidents.

(2) When the identity becomes known this is substituted in the hospital records ("Peter Jones-AKA unknown male Foxtrot 23/4") while the hospital admission number is retained and is used in all laboratory and radiography data.

(3) Hospital computer searches for people seeking relatives or other agencies has been simplified with inquiries based on either the term "unknown" or probable date of admission.

(4) Although a few other systems of alias assignment have been suggested in the literature, ${ }^{12}$ they do not enhance the staff's ability to communicate the identity, are limited by the number of alias names available, and difficulty of computer searches.

We advocate this system as a simple yet effective, tried and tested method for the rapid assignment of a unique alias to critically ill or injured unknown patients.

Conflict of interest: none.

Funding: none.

1 Robinson G, Fortune JB, Wachtel TL, et al. A system of alias assignment for unidentified patients requiring emergency hospital admission. $\mathcal{f}$ Trauma $1985 ; 25: 333-6$.

2 Blank-Reid CA, Kaplan LJ. A system for working with unidentified trauma patients. International fournal of Trauma Nursing 1996;2:108-10.

\section{Faculty of Accident and Emergency Medicine}

Consultant appointments, September to December 1998

Gary Cumberbatch
Ian Stell
Sean McGovern
Clifford Mann
Dyfrig Hughes
Daniel Wallis
Fiona Barratt
Julian Webb
George Little
James Steele
Seamus O'Reilly
Mark Prescott
Gerard Lane
Ian Greaves

Gary Cumberbatch

Clifford Mann

Dyfrig Hughes

Daniel Wallis

Fiona Barratt

James Steele

Seamus O'Reilly

Gerard Lane

Ian Greaves

\author{
Poole Hospital, Dorset \\ Farnborough Hospital, Kent \\ The Ulster Hospital, Northern Ireland \\ Taunton and Somerset Hospital \\ Barnsley District General Hospital \\ St George's Hospital, Tooting \\ St Richard's Hospital, Chichester \\ Kent and Sussex Hospital \\ King's College Hospital, London \\ Altnagelvin Hospital, Northern Ireland \\ Craigavon Area Hospital, Northern Ireland \\ Royal Shrewsbury Hospital \\ Southend Hospital \\ Royal Defence Medical College
}

